\title{
A reference material for dynamic displacement calibration
}

\author{
Andrea Davighi ${ }^{1}$, Erwin Hack $^{2}$, Eann Patterson ${ }^{3 a}$, Maurice Whelan ${ }^{1}$ \\ ${ }^{1}$ Institute for Health and Consumer Protection, European Commission DG Joint Research Centre, \\ 21021 Ispra (VA), Italy \\ ${ }^{2}$ EMPA, Swiss Federal Laboratories for Materials Testing and Research, Laboratory for \\ Electronics/Metrology/Reliability, CH-8600 Dübendorf, Switzerland \\ ${ }^{3}$ Composite Vehicle Research Center, Michigan State University, East Lansing, MI, USA
}

\begin{abstract}
Calibration of displacement and strain measurement systems is an essential step in providing traceability and confidence in stress and strain distributions obtained from experiment and used to validate simulations employed in engineering design. Reference materials provide a simple, well-defined distribution of the measured quantity that can be traced to an international standard and can be used to assess the uncertainty associated with the measurement system. Previous work has established a reference material and procedure for calibrating optical systems for measuring static, in-plane strain distributions and also demonstrated its use. A new effort is in progress to extend this work to the measurement of three-dimensional displacement distributions induced by cyclic and dynamic loading, including transients and large-scale deformation. The first step in this effort has been to define both the essential and desirable attributes of a reference material for calibrating systems capable of measurements of dynamic displacement and strain. An international consortium of research laboratories, system designers, manufacturers and end-users has identified a list of attributes and members of the experimental mechanics community have been asked to weight the importance of these attributes. The attributes are being utilised to evaluate candidate designs for the reference material which have been generated through a series of brain-storming sessions within the consortium.
\end{abstract}

\section{Introduction}

\subsection{Engineering context}

Arguably the engineering profession is facing the greatest challenge in its history in assisting society to 'avoid the unmanageable and to manage the unavoidable' [1] in terms of the effects of global climate change. Current engineering solutions, both in terms of products and services, are not sustainable and 'new' solutions need to be found quickly. In a nut-shell the 'equivalent of 200 years of development is needed in less than 40 years' [2] since as a consequence of indulging in

a email: eann@egr.msu.edu 
'thermodynamic whoopee with fossil fuels' [3] we have 'all but destroyed this once salubrious planet as a life-support system in fewer than two hundred years' [3]. There is much attention paid to finding alternative sources of energy that are renewable or sustainable and there is significantly less focus on efficient use of energy to reduce our dependence on fossil fuels. Perhaps the redesign of existing devices and means of transportation so that they consume substantially less energy is less exciting or glamorous than developing new energy sources. Efficiency attracts less plaudits than discovery but requires a comparable level of ingenuity and attention to detail for comparable gains.

By definition energy is closely related to work and hence to forces and distance moved, thus in order to reduce energy consumption by machines, in general, one must either reduce the distances moved or the force required to do the moving. Since the force is usually required to overcome inertia reducing mass is an effective way of reducing energy consumption. However, mass is often associated with material that provides structural integrity and so mass reduction cannot be at the expense of safety which would be unacceptable to society. The engineering solution is to use the mass of material more efficiently to provide structural integrity either through ingenious geometric design or through deploying advanced materials that are stronger and lighter. In both cases more sophisticated structural modelling is usually required compared to the original design as a consequence of the more complex geometry and, or material.

\subsection{Validation of structural analyses}

The more sophisticated structural analyses, referred to above, stretch the boundaries of engineering knowledge and hence a rigorous process of verification and validation is necessary in order to manage the associated risks and ensure safety. Schwer [4] has identified the key components of a verification and validation process. Verification consists of eradicating programming and logic errors from the code being employed in the computational analysis and then estimating errors associated with the modelling assumptions and discretization. Validation is evaluating the extent to which the computational model is an accurate representation of the real world, at least from the perspective of the planned purposes of the model. Validation should involve the conduct of experiments specifically designed to provide a comparison with the real world though it needs to be recognised that most experiments are also a representation of the real world so engineers often resort to prototype devices used in service conditions as sources of data.

A crucial part of the validation process is comparing data from an experiment with data from the computational model. It is common practice to compare data at single points, usually the stress 'hot-spot' identified in the model and at which location a strain gauge has been bonded in the experiment. While useful, this approach is very limiting when it is desired to refine a design by removing a substantial mass of material or when using a damage tolerant approach to design against fatigue. Optical techniques for strain measurement allow strain maps to be obtained over a wide field of view and consequently offer the potential for a more rigorous validation process. Recent work [5] has established a reference material for static, in-plane strain measurement and demonstrated [6] a protocol for its use that allows a minimum level of uncertainty to be established.

While the establishment of a reference material and accompanying protocol for static analyses is a valuable step, two important gaps remain: an effective means of quantitatively comparing large data fields as part of the validation process, e.g. data fields from experiment and from computational modelling; and the capability to calibrate optical systems for displacement or strain measurement under non-static conditions, i.e. cyclic, dynamic or transient conditions. The filling of these gaps are amongst the goals of the ADVISE project (Advanced Dynamic Validation through Integration of Simulations and Experimentation).

\subsection{The ADVISE project}

The ADVISE (Advanced Dynamic Validation through Integration of Simulations and Experimentation) project [7] is a three-year program partly funded by the EU Framework 7 which 
began in late 2008. As with its predecessor, the SPOTS project [8], the ADVISE project was conceived to span the innovation process from research laboratories to instrument manufacturers and suppliers to end-users in a range of industries. The partners are Airbus (UK), Centro Ricerche Fiat (Italy), Dantec Dynamics GmbH (Germany), EC Joint Research Centre, EMPA Swiss Federal Laboratories for Materials Testing and Research (Switzerland), High Performance Space Structure Systems GmbH (Germany), Michigan State University (USA), University of Liverpool (UK) and the University of Patras (Greece).

\section{Design of the reference material}

\subsection{Specification}

The two goals identified above of designing a reference material for calibrating optical systems capable of measuring non-static strain and, or displacement distributions, and of developing the capability to quantitatively compare large maps of strain tensors, are being tackled in parallel. Here the approach to designing the reference material is reported and the quantitative comparison of data maps is described elsewhere [9].

At the most fundamental level the reference material has to provide the first link in the chain of traceability to an international standard. In this case, the length standard was chosen as being most appropriate to displacement and strain measurement since both are derived from length. In addition, this was the standard selected for the static, in-plane reference material $[5,6]$. As with the static, inplane reference material, it must be possible to scale the new reference material to allow calibrations and subsequently validations to be performed at whatever scale is appropriate to the service application. The extension of the design specification relative to that considered by the SPOTS project was to consider three-dimensional motion at velocities and accelerations encountered in cyclic loading applications and single high-speed events both of the linear and non-linear type in which the displacements and deformations are geometrically and materially non-linear with respect to the applied load. In the ADVISE project these three types of events have been termed: 'cyclic', 'transient' and 'non-linear'. It is desirable that a single reference material should be designed to allow calibration for all three types of loading and this is the premise from which the design process has started.

\subsection{Rational Decision Making Process}

The design specification described above is very challenging and so the rational decision making model [10] is being employed to guide the process. In this model attributes that it is required and, or desired for the design to possess are identified and weighted according to importance. Then candidate designs are generated, often via a brainstorming process, and are subsequently ranked against the attributes by evaluating the extent to which they possess each attribute and multiplying by the appropriate weighting. The rank order of candidate designs is used to select a final design or pair of designs for embodiment and refinement. This process allows a controlled and objective exploration of a large design space thus increasing the probability of identifying a close to ideal design concept which can be optimised though a more usual focussed and prosaic design process.

To refine the design specification described above and to identify the attributes required and desired for the dynamic reference material a group from the ADVISE project met in Sheffield, UK in March 2009. One key decision made at this meeting was that the three-dimensional displacement field in the reference material should be the fundamental quantity considered since both strain and stress tensors are derived from this quantity. After the meeting, all participants in the project weighted the identified attributes in terms of relative importance on a scale of 1 (unimportant) to 5 (essential). Subsequently the project concept, design specification and attributes were presented at a workshop organised by VAMAS Technical Working Area 26 [11] at the Society for Experimental 
Mechanics Spring Conference in Albuquerque NM in June 2009. Participants at the workshop were asked to weight the attributes and afterwards all members of the Society for Experimental Mechanics were invited by email to participate in weighting the attributes and proposing attributes that they felt had been omitted. The results are shown in figures 1 and 2 divided into those attributes associated with the displacement field to be generated and measured in the reference material and those that the physical reference material should possess.

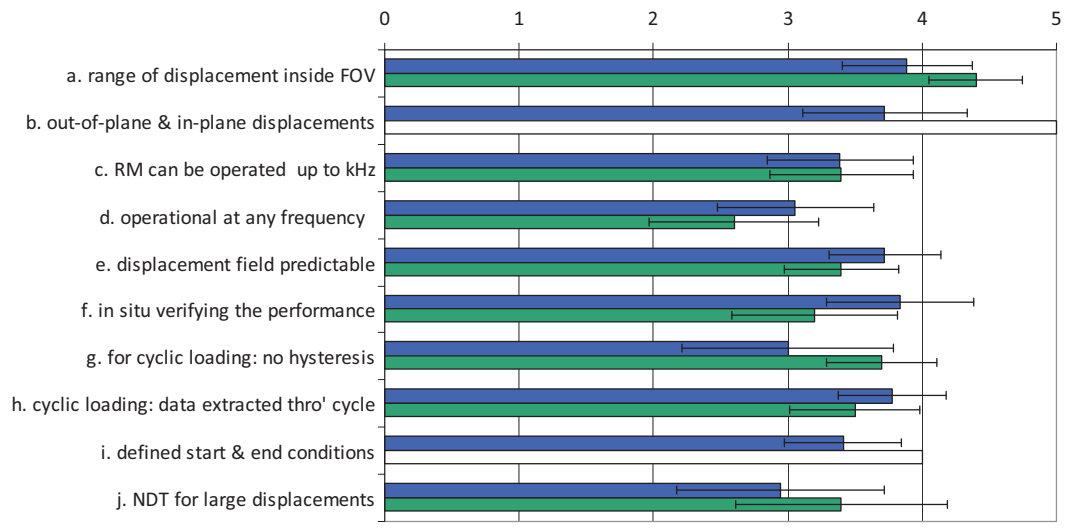

Fig. 1. Attributes and their weightings for the displacement field in the dynamic reference material. The weightings (1 - unimportant, 2 - preferred, 3 - important, 4 - highly desirable, or 5 - essential) assigned by individual partners in the project were averaged (green) and the same exercise repeated for the wider community (blue). Some attributes were suggested by the community during the weighting process (white) and so were not weighted by a significant number of participants. (@ADVISE consortium)

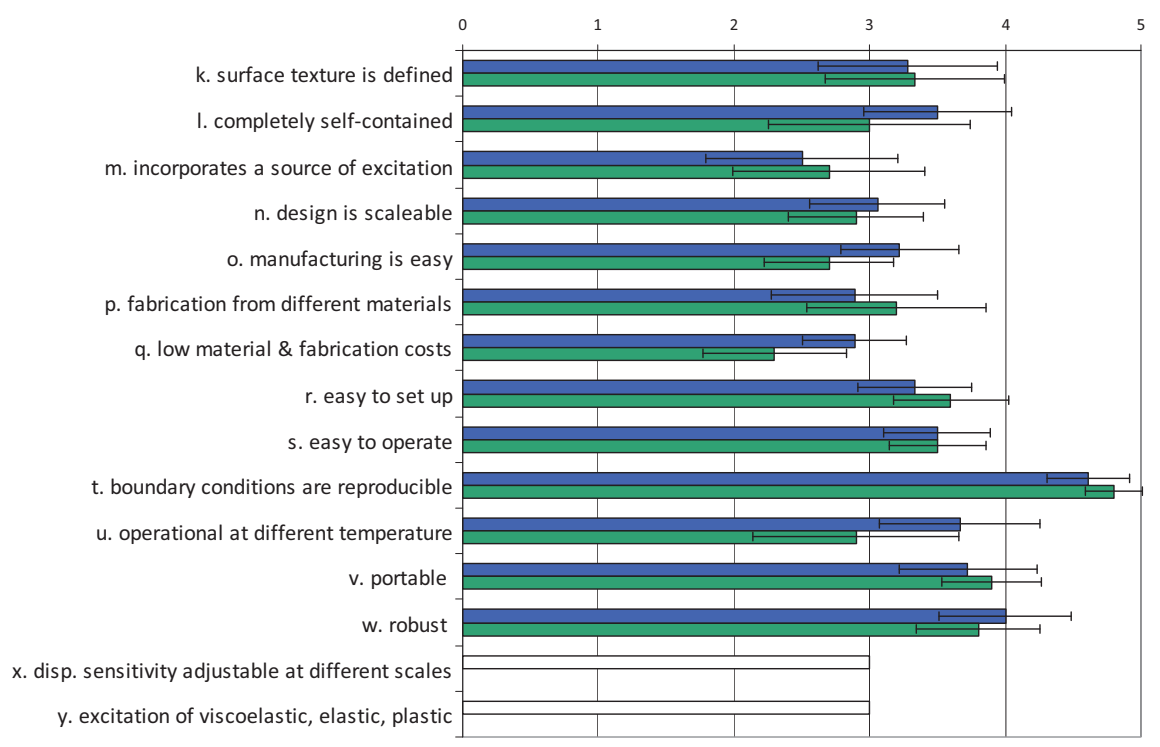

Fig. 2. Attributes and their weightings for the physical embodiment of the dynamic reference material. The weightings ( 1 - unimportant, 2 - preferred, 3 - important, 4 - highly desirable, or 5 - essential) assigned by individual partners in the project were averaged (green) and the same exercise repeated for the wider community (blue). Some attributes were suggested by the community during the weighting process (white) and so were not weighted by a significant number of participants. (CADVISE consortium) 
The essential attributes have been selected to be those for which in the combined results from the community and the consortium the mean weighting plus one standard deviation was larger than 4.0. As a result the essential attributes are:

a. there is a range of displacement values inside the field of view

b. in-plane and out-of-plane displacements available

$\mathrm{f}$. there is a means of verifying the performance in situ

h. for cyclic loading: data can be extracted throughout the cycle

t. the boundary conditions are reproducible

v. it is portable

w. it is robust

In September 2009 the ADVISE consortium met in Ispra, Italy to brainstorm a set of candidate designs for the dynamic reference material. These designs were tested against the essential attributes and those that did not possess all of the essential attributes were rejected. At the time of writing the process of assessing the remaining, viable candidate designs against the desirable attributes is being undertaken. This is not a straightforward process and involves some experimentation and simulation of candidate designs to establish their performance characteristics.

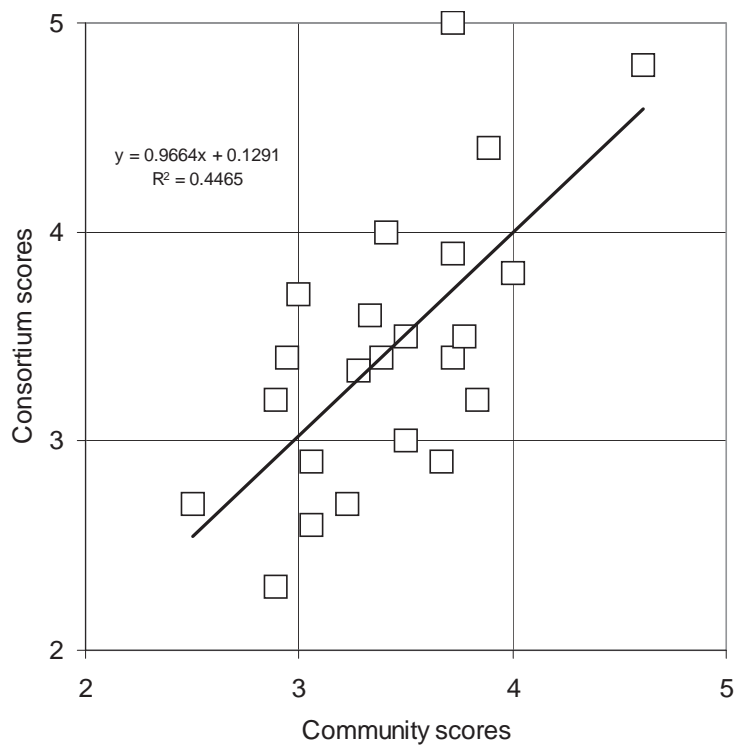

Fig. 3 Correlation of the weightings by the ADVISE consortium partners and the members of the scientific community of the attributes required or desired in the dynamic reference material. (C) ADVISE consortium)

\section{Discussion and Conclusions}

The approach described above was successfully employed in the SPOTS project which with hindsight might seem straightforward compared to the ADVISE specification but at its instigation represented a very substantial and challenging step into the unknown. The process has a significant advantage in that it allows the involvement of the wider scientific community which is seen as essential in order to ensure the acceptance of the reference material design and protocol that ultimately it is hoped will form part of a standard. It is encouraging to observe that there is a high degree of correlation between the views of the community at large and of the members of the ADVISE consortium as shown in figure 3. This confirms that the selection of consortium members 


\section{EPJ Web of Conferences}

was appropriate at least in terms of representing the views of the community. Successful completion of the project on schedule in late 2011/early 2012 will confirm whether the selection was appropriate in terms of the skill set required for the undertaking!

The intention is to complete the design of the reference material, develop the protocol for its use in the calibration of optical systems for measuring dynamic displacements and evaluating the resultant strains and stresses, and to combine this work with the parallel effort to develop methodologies for quantitatively comparing maps of strain and, or stress tensors [9]. The whole body of work will then be incorporated into a revision of the guidelines for calibration and evaluation produced by the earlier SPOTS project [8].

\section{Acknowledgements}

The authors cheerfully acknowledge the inputs of the following persons in the ADVISE project: Richard Burguete, Mara Feligiotti, Alexander Ihle, George Lampeas, John Mottershead, Andrea Pipino, Hans Reinhard Schubach, Thorsten Siebert, and Victor Wang. The ADVISE project is a Seventh Framework Programme Collaborative Project within Theme 7: Transportation including Aeronautics (Grant no. 218595).

\section{References}

1. Thomas L. Friedman, Hot, Flat and Crowded - Why we need a green revolution and how it can renew America (Farrar, Straus \& Giroux, New York, 2008)

2. Yoshiyuki Sakaki, Keynote presentation at ICEE/ICEER conference in Seoul, Korea, 25th August 2009

3. Kurt Vonnegut, A Man Without a Country (Bloomsbury, London, 2006)

4. L.E. Schwer, Engineering with Computers, 23:245-252 (2007)

5. E.A. Patterson, E. Hack, P. Brailly, R.L. Burguete, Q. Saleem, T. Seibert, R.A. Tomlinson, M.P. Whelan, Optics and Lasers in Engineering, 45:550-564 (2007)

6. M.P. Whelan, D. Albrecht, E. Hack, E.A. Patterson, Strain, 44:180-190(2008)

7. www.dynamicvalidation.org

8. www.opticalstrain.org

9. J. Mottershead, A. Patki, E.A. Patterson, V. Wang, Image decomposition as a tool for validating stress analysis models, Proc. $14^{\text {th }}$ Int. Conf. on Experimental Mechanics, Poitiers, France, $4-8^{\text {th }}$ July, 2010, paper no. 271.

10. N. Cross, Engineering Design Methods (John Wiley \& Sons, London, 1989)

11. www.twa26.org 